\title{
Comparative performance of Eucalyptus bicostata, E. globulus, E. maidenii and E. pseudoglobulus on three northern New Zealand sites
}

\author{
Desmond J Stackpole ${ }^{1}$, Ruth M McConnochie ${ }^{2}$, Heidi S Dungey ${ }^{1}$, Charlie B Low ${ }^{1}$, Rowland D Burdon \\ and Stuart G Kennedy ${ }^{1 *}$
}

\begin{abstract}
Trials to compare eucalypt species from the blue gum group, including Eucalyptus bicostata Maiden, Blakely \& Simmons, E. globulus Labill., E. maidenii F. Muell., and E. pseudoglobulus Naudin ex Maiden were established in 1999 at three sites in the North Island of New Zealand (Northland, Bay of Plenty, and Hawke's Bay). Each species was planted out in replicated 49-tree plots consisting of seven individuals from seven provenances. Only two provenances of E. pseudoglobulus were available and these were outcompeted by inter-planted E. globulus so were not formally assessed. Measurements taken at age seven years indicate a strong species $\times$ site effect for foliage health, stem straightness and survival. Generally, growth and foliar health were best at the Hawke's Bay site. Provenance $x$ site interaction was mostly absent for each of the blue gum species. However, there was a significant provenance variation for most traits within E. bicostata, and provenance $\mathrm{x}$ site interaction; both of which were largely driven by the performance of the northernmost Wollemi provenance. While some interactions occurred at the species $\times$ site level, E. maidenii performed consistently well in growth, stem straightness and health compared with the other taxa. Eucalyptus maidenii appears to be the fastest growing of the species tested within the broad region covered by the study sites, in the warmer coastal areas of New Zealand's North Island.
\end{abstract}

Keywords: Eucalyptus bicostata; Eucalyptus globulus; Eucalyptus maidenii; Eucalyptus pseudoglobulus provenance trials; Species x site trials; Provenance $x$ site interaction; Growth rate; Straightness; Foliage health; New Zealand

\section{Background}

The blue gum group comprises four closely related taxa that are variously treated as separate species Eucalyptus bicostata Maiden, Blakely \& Simmons, E. globulus Labill., E. maidenii F. Muell., and E. pseudoglobulus Naudin ex Maiden (Brooker, 2000) or as subspecies of E. globulus (Jordan et al., 1993; Kirkpatrick, 1975). For this paper, they are treated as separate species. The species complex appears to have radiated from an origin in eastern Victoria (Jones, 2002), with well-defined core populations (Figure 1), and extensive intergrades between species at the boundaries. Eucalyptus globulus has the most southerly distribution of the four species, occurring in Tasmania, the Bass Strait Islands and the southern extremities of eastern Victoria (Table 1). The core

\footnotetext{
* Correspondence: stuart.kennedy@scionresearch.com

'Scion, 49 Sala Street, Private Bag, 3020, Rotorua, New Zealand

Full list of author information is available at the end of the article
}

distributions of the other blue gums are more northerly than that of E. globulus (Figure 1). Of these, E. pseudoglobulus intergrades with E. globulus, E. bicostata and E. maidenii (Jones, 2002), from its east Victorian core. Eucalyptus bicostata occurs on the inland watershed of Victoria and southeast New South Wales, is geographically separated from other species, occupies a substantial latitudinal range, and has extreme outlying populations such as Mount Bryan (South Australia) (Vaillancourt et al., 2001), and Wollemi (New South Wales). Over this range, two rainfall distribution zones are recognised: (1) predominantly winter rainfall in the south; and (2) uniform annual rainfall at Wollemi ${ }^{\mathrm{a}}$. Also, frosts are more common and severe in the south. The range of E. maidenii occurs on the coastal catchments of south-eastern New South Wales, which experiences a more uniform rainfall distribution (Bureau of Meteorology 2012). 


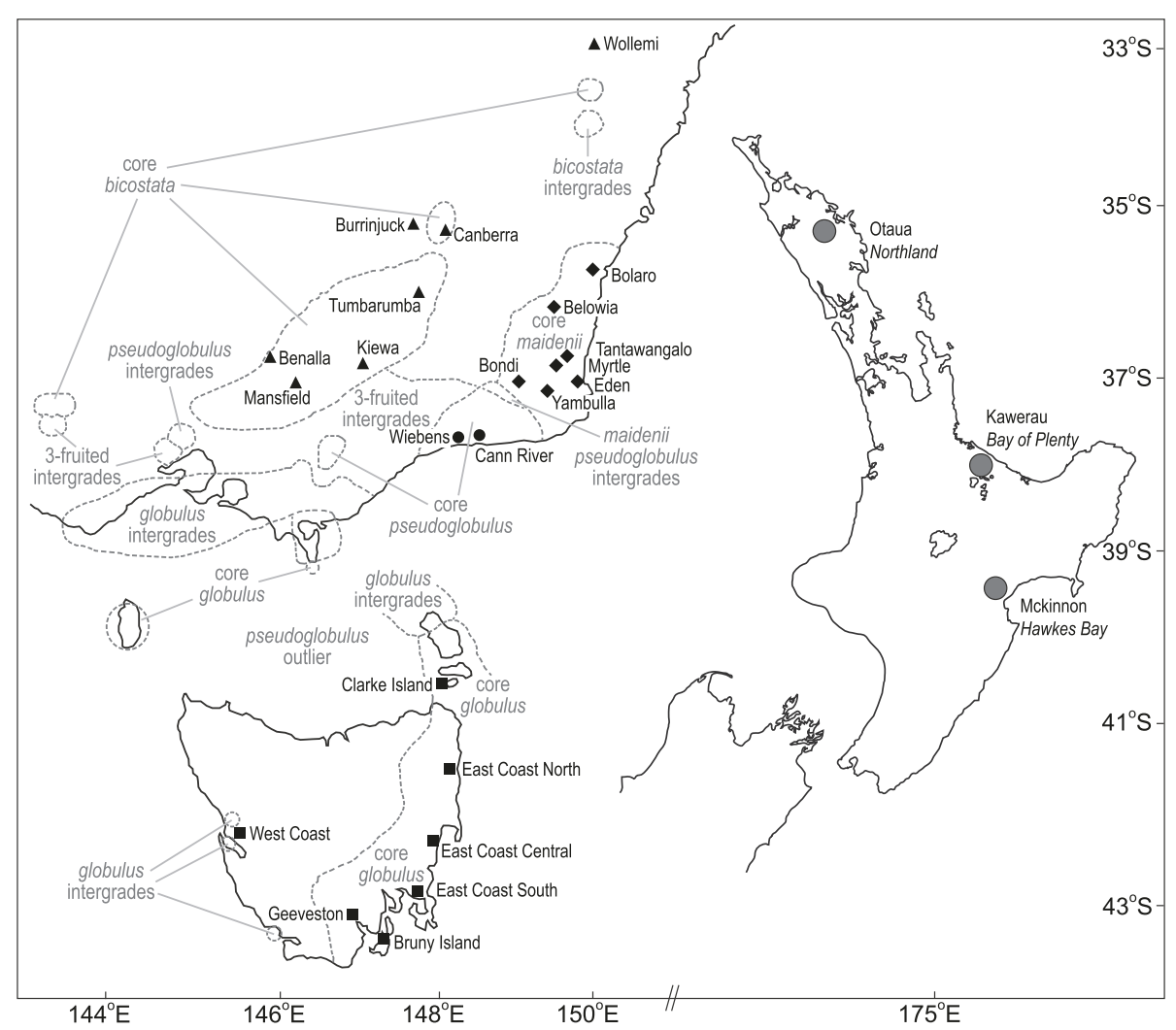

Figure 1 Collection origins of the blue gum provenances $\bullet=E$. maidenii; $\mathbf{\|}=$ E. globulus; $\boldsymbol{\Delta}=E$. bicostata; $\bullet=E$. pseudoglobulus; $\mathrm{O}=\mathrm{s}$ mall local population; $\mathrm{X}=$ locations of the three New Zealand test sites in Northland (Otaua) Bay of Plenty (Kawerau) and Hawkes Bay (McKinnon), overlain on the locations of core populations of each species (Redrawn from Potts et al., 2004).

Eucalyptus globulus, along with its intergrades with $E$. pseudoglobulus, has proved suited to both kraft pulp production (Kibblewhite et al., 2000), and solid wood products (Boland et al., 1992); and together have formed the genetic base of large-scale plantations across xeric, temperate, winter-maximum rainfall landscapes in the Iberian Peninsula, Australia and South America (Lanfranco \& Dungey, 2001; Potts et al., 2004; Díez 2005). Species × provenance trials in Tasmania (Volker \& Orme, 1988) and Portugal (Almeida et al., 1995) confirm the superiority of E. globulus over the other blue gum species at sites with a dry summer climate but with good winter rainfall. However, different specific rankings have been reported from tests in warmer climates with more uniform seasonal distribution of rainfall. These include: anecdotal reports from central Africa and southern China (Eldridge et al., 1993); and formal comparisons among the species in northern New Zealand (Low \& Shelbourne, 1999) and South Africa (Komakech et al., 2009). When compared in ex-situ species comparison tests, the relative performance of these species is expected to be related to the fit between the environmental parameters of the collection and test sites, and such relationships have been considered in planning introduction trials (e.g. Booth, 2005).

Variations in disease resistance (Hood et al., 2002), wood chemical composition (Rencoret et al., 2007), and pulp yield (Kibblewhite et al., 2000) have been demonstrated among species. Genetic variation within and among subraces, which has been demonstrated in many $E$. globulus traits, is likely to be similarly exploitable within

Table 1 Natural distribution and range of climatic conditions of the four blue gum species in Australia extracted from Boland et al., (1992)

\begin{tabular}{|c|c|c|c|c|c|c|c|}
\hline Species & $\begin{array}{l}\text { Latitude } \\
\text { range }\left({ }^{\circ} \mathrm{S}\right)\end{array}$ & $\begin{array}{l}\text { Elevation } \\
(\mathrm{m})\end{array}$ & $\begin{array}{l}\text { Min. Mean coldest } \\
\text { month, }\left({ }^{\circ} \mathrm{C}\right)\end{array}$ & $\begin{array}{l}\text { Max. Mean hottest } \\
\text { month, }\left({ }^{\circ} \mathrm{C}\right)\end{array}$ & $\begin{array}{l}\text { Mean annual } \\
\text { rainfall (mm) }\end{array}$ & $\begin{array}{l}\text { Distribution of } \\
\text { rainfall }^{1}\end{array}$ & $\begin{array}{l}\text { Number of } \\
\text { frosts per year }\end{array}$ \\
\hline E. maidenii & $34.7-37.5$ & $200-900$ & $-4-+2$ & $+23-+27$ & $800-1200$ & U & $20-80$ \\
\hline E. globulus & $38.5-43.5$ & $0-450$ & $-0-+8$ & $+20-+23$ & $600-1400$ & WS & $5-40$ \\
\hline E. bicostata & $31.0-38.0$ & $0-1050$ & $-2-+5$ & $+25-+32$ & $700-1200$ & WS south, U north & $25-70$ \\
\hline E. pseudoglobulus & $37.0-40.0$ & $0-100$ & $+2-+4$ & $+24-+27$ & $700-1100$ & slight WS & $1-15$ \\
\hline
\end{tabular}

${ }^{1} \mathrm{WS}=$ most rainfall in winter and spring, $\mathrm{U}=$ seasonal rainfall uniformly distributed. 
the other three blue gum species (Dutkowski \& Potts, 1999; Jordan et al., 1993; Nesbitt et al., 1995; Potts et al., 2004). This has already been reported for disease resistance in young E. bicostata (Hood et al., 2002).

The wood of the blue gum complex is recognised for its suitability for producing chemical pulps (Cotterill \& Brolin, 1997; Kibblewhite et al., 2000; Miranda \& Pereira, 2001), and has higher density, modulus of elasticity, modulus of rupture, and lower shrinkage than that of E. nitens (Deane \& Maiden) Maiden, a common plantation alternative (McKinley et al., 2002). In New Zealand, research has shown that $E$. maidenii matched E. globulus in growth and foliage health (Low \& Shelbourne 1999). Although the pulp yield of $E$. maidenii, was lower (Kibblewhite et al., 2000), its density was higher by some $50 \mathrm{~kg} \mathrm{~m}^{-3}$ (Jamieson, 1997).

In 1999, a series of species / provenance trials were established in New Zealand, designed to assess the potential of E. bicostata, E. globulus, E. maidenii and E. pseudoglobulus, and to gain insight into the effects of different provenances of these species across three sites in New Zealand that were located within the equivalent range of the Australian populations. This paper presents and discusses the results of growth, form and health assessments of these trials at age seven years.

\section{Methods}

Species $\times$ provenance trials containing E. bicostata, E. globulus, E. maidenii and E. pseudoglobulus were established at three North Island sites in the winter of 1999, one each at Otaua, Northland; Kawerau, Bay of Plenty; and McKinnon, Hawke's Bay (Table 2, Figure 1). The trials were set out as a randomised complete block design, with 4 to 8 replicates per site ( 4 at McKinnon, 5 at Otaua and 8 at Kawerau). Replicates consisted of five, 49-tree (7 trees $\times 7$ trees) blocks. The species blocks for E. bicostata, E. globulus and E. maidenii each contained seven seedlings from seven provenances of that species, arranged at random within the block. However, only two provenances of E. pseudoglobulus were present so these were mixed with five provenances of E. globulus to make up the fourth block. Provenance details for each species are provided in Table 3. The fifth block contained; eight control seedlots, four of $E$. nitens (bulked across Toorongo (Victoria), various New South Wales provenances and the Waikuku land race from New Zealand) and four of E. globulus. There were six trees per seedlot and a seventh tree for one of the controls to make up the 49-tree block.

The stocking at each trial was 1114 stems per hectare, equivalent to $3.5 \mathrm{~m}$ between rows and $3 \mathrm{~m}$ within rows. McKinnon was an ex-pasture site, with a 2-year-old crop of pine which was pre-sprayed with glyphosphate $\left(10 \mathrm{~L} \mathrm{ha}^{-1}\right)$ by helicopter. The area was also fenced off at the time of planting due to concern about damage from wild goats. The trial area was spot-mounded. Otaua was an ex-pasture site that was spot-cultivated using a Savanah Cultivator head (spot size: $1.20 \mathrm{~m}$ ) to a depth of $60 \mathrm{~cm}$. A mixture of glyphosphate $6 \mathrm{~L} \mathrm{ha}^{-1}+$ terbuthylazine $20 \mathrm{~L} \mathrm{ha}^{-1}$ was applied to each spot at a rate of $30 \mathrm{~mL}$. Kawerau was on an ex-pasture site prepared by ripping and mounding and a pre-plant application of terbuthylazine $6 \mathrm{~L} \mathrm{ha}^{-1}+$ glyphosphate $4 \mathrm{~L} \mathrm{ha}^{-1}$ + organo-silicone penetrant $500 \mathrm{~mL} \mathrm{ha}^{-1}$, water rate $150 \mathrm{~L} \mathrm{ha}^{-1}$.

Traits measured were:

- Diameter at Breast Height (DBH) measured at $1.4 \mathrm{~m}$ above ground level $(\mathrm{cm})$,

- Height (HT) from ground level to the growing tip $(\mathrm{m})$,

- Stem straightness (STR), 1-9 scale where 1 = most crooked and $9=$ straightest),

- Foliage health (FH), 0-9 scale ${ }^{\mathrm{b}}$, where $0=\operatorname{dead}$ or dying and 9 = healthy,

- Green crown height (GCH), height from ground level to the base of the green crown, defined as the point of extension of the lowest foliage-bearing branch from stem (m),

- Survival (SUR), dead [0] or alive [1].

At age 7 years, the inner 25 trees of the original 49 trees planted per block were measured. The inter-planted E. globulus seedlots rapidly suppressed the two E. pseudoglobulus entries, which were

Table 2 Location and climate of the three trial sites in the North Island, New Zealand

\begin{tabular}{|c|c|c|c|c|c|c|c|c|c|c|c|}
\hline Site & Region & $\begin{array}{l}\text { Latitude } \\
\left.\text { ( }{ }^{\circ} \mathrm{S}\right)\end{array}$ & $\begin{array}{l}\text { Longitude } \\
\left({ }^{\circ} \mathrm{E}\right)\end{array}$ & $\begin{array}{l}\text { Elevation } \\
(\mathrm{m})\end{array}$ & $\begin{array}{l}\text { Min. Mean } \\
\text { coldest } \\
\text { month }{ }^{1} \\
\left({ }^{\circ} \mathrm{C}\right)\end{array}$ & $\begin{array}{l}\text { Max. Mean } \\
\text { hottest } \\
\text { month }{ }^{1} \\
\left({ }^{\circ} \mathrm{C}\right)\end{array}$ & $\begin{array}{l}\text { Distribution } \\
\text { of rainfall }^{1,2}\end{array}$ & $\begin{array}{l}\text { Number of } \\
\text { frosts per year }{ }^{1}\end{array}$ & $\begin{array}{l}\text { Mean } \\
\text { annual } \\
\text { rainfall }^{1} \\
(\mathrm{~mm})\end{array}$ & $\begin{array}{l}\text { Slope } \\
\left({ }^{\circ}\right)\end{array}$ & $\begin{array}{l}\text { Soil } \\
\text { type }\end{array}$ \\
\hline Otaua & Northland & $35^{\circ} 30^{\prime}$ & $173^{\circ} 40^{\prime}$ & 180 & 11 & 19 & U & 0 & 1800 & 15 & $\begin{array}{l}\text { Clay loam } \\
\text { pasture }\end{array}$ \\
\hline Kawerau & $\begin{array}{l}\text { Bay of } \\
\text { plenty }\end{array}$ & $38^{\circ} 05^{\prime}$ & $176^{\circ} 42^{\prime}$ & 200 & 9 & 19 & U & 12 & 1750 & $<10$ & Pumice \\
\hline McKinnon & $\begin{array}{l}\text { Hawkes } \\
\text { Bay }\end{array}$ & $39^{\circ} 15^{\prime}$ & $177^{\circ} 00^{\prime}$ & 10 & 11 & 17 & U & 0 & 1500 & $<10$ & $\begin{array}{l}\text { Fertile } \\
\text { bottomland }\end{array}$ \\
\hline
\end{tabular}

\footnotetext{
${ }^{1}$ These data were provided by National Institute of Water and Atmospheric Research (2011) on a $500 \mathrm{~m}$ raster grid.
}

${ }^{2} \mathrm{WS}=$ most rainfall in winter and spring, $\mathrm{U}=$ seasonal rainfall uniformly distributed. 
Table 3 Collection locations of provenances of Eucalyptus maidenii, E. globulus, E. bicostata and E. pseudoglobulus used in this study

\begin{tabular}{|c|c|c|c|c|c|c|}
\hline Species & Provenance $^{1}$ & Australian State $^{2}$ & Latitude (S) & Longitude (E) & Elevation (m) & Seedlot code \\
\hline E. maidenii & $\begin{array}{l}\text { Bolaro Mt. } \\
\text { Belowra } \\
\text { Tantawangalo } \\
\text { Myrtle Mt. } \\
\text { Eden } \\
\text { Bondi SF } \\
\text { Yambulla SF }\end{array}$ & $\begin{array}{l}\text { NSW } \\
\text { NSW } \\
\text { NSW } \\
\text { NSW } \\
\text { NSW } \\
\text { NSW } \\
\text { NSW }\end{array}$ & $\begin{array}{l}35^{\circ} 40^{\prime} \\
36^{\circ} 10^{\prime} \\
36^{\circ} 54^{\prime} \\
36^{\circ} 52^{\prime} \\
37^{\circ} 04^{\prime} \\
37^{\circ} 08^{\prime} \\
37^{\circ} 12^{\prime}\end{array}$ & $\begin{array}{l}150^{\circ} 02^{\prime} \\
149^{\circ} 43^{\prime} \\
149^{\circ} 38^{\prime} \\
149^{\circ} 40^{\prime} \\
149^{\circ} 52^{\prime} \\
149^{\circ} 17^{\prime} \\
149^{\circ} 28^{\prime}\end{array}$ & $\begin{array}{l}380 \\
340 \\
540 \\
480 \\
- \\
- \\
480\end{array}$ & $\begin{array}{l}\text { CSIRO } 18728 \\
\text { CSIRO } 19456 \\
\text { CSIRO } 19460 \\
\text { CSIRO } 19458 \\
\text { AmCor } 3499 \\
\text { CSIRO } 19454 \\
\text { CSIRO } 19284\end{array}$ \\
\hline E. globulus & $\begin{array}{l}\text { Clarke Island } \\
\text { EC North } \\
\text { WC Central } \\
\text { EC Central } \\
\text { EC South Central } \\
\text { Geeveston } \\
\text { Bruny Island }\end{array}$ & $\begin{array}{l}\text { Tas. } \\
\text { Tas. } \\
\text { Tas. } \\
\text { Tas. } \\
\text { Tas. } \\
\text { Tas. } \\
\text { Tas. }\end{array}$ & $\begin{array}{l}40^{\circ} 31^{\prime} \\
41^{\circ} 19^{\prime} \\
42^{\circ} 00^{\prime} \\
42^{\circ} 09^{\prime} \\
42^{\circ} 32^{\prime} \\
43^{\circ} 10^{\prime} \\
43^{\circ} 09^{\prime}\end{array}$ & $\begin{array}{l}148^{\circ} 10^{\prime} \\
148^{\circ} 15^{\prime} \\
145^{\circ} 20^{\prime} \\
148^{\circ} 03^{\prime} \\
147^{\circ} 57^{\prime} \\
146^{\circ} 55^{\prime} \\
147^{\circ} 20^{\prime}\end{array}$ & $\begin{array}{l}- \\
- \\
- \\
- \\
- \\
- \\
-\end{array}$ & $\begin{array}{l}\text { NFP1 } \\
\text { NFP2 } \\
\text { NFP5 } \\
\text { NFP3 } \\
\text { NFP4 } \\
\text { CSIRO } 18723 \\
\text { CSIRO18726 }\end{array}$ \\
\hline E. bicostata & $\begin{array}{l}\text { Canberra } \\
\text { Burrinjuck } \\
\text { Tumbarumba } \\
\text { Wollemi } \\
\text { Benalla } \\
\text { Kiewa } \\
\text { Mansfield }\end{array}$ & $\begin{array}{l}\text { ACT } \\
\text { NSW } \\
\text { NSW } \\
\text { NSW } \\
\text { Vic. } \\
\text { Vic. } \\
\text { Vic. }\end{array}$ & $\begin{array}{l}35^{\circ} 19^{\prime} \\
35^{\circ} 02^{\prime} \\
35^{\circ} 46 \\
32^{\circ} 59^{\prime} \\
36^{\circ} 46^{\prime} \\
36^{\circ} 48^{\prime} \\
37^{\circ} 05^{\prime}\end{array}$ & $\begin{array}{l}147^{\circ} 08^{\prime} \\
148^{\circ} 40^{\prime} \\
148^{\circ} 04 \\
150^{\circ} 23^{\prime} \\
145^{\circ} 50^{\prime} \\
147^{\circ} 13^{\prime} \\
146^{\circ} 18^{\prime}\end{array}$ & $\begin{array}{l}- \\
- \\
- \\
- \\
- \\
-\end{array}$ & $\begin{array}{l}\text { Amcor } 239 \\
\text { Amcor } 242 \\
\text { Amcor } 238 \\
\text { Amcor } 243 \\
\text { Amcor } 244 \\
\text { Amcor } 235 \\
\text { Amcor } 245\end{array}$ \\
\hline E.pseudoglobulus & $\begin{array}{l}\text { Wiebens } \\
\text { Cann River }\end{array}$ & $\begin{array}{l}\text { Vic. } \\
\text { Vic. }\end{array}$ & $\begin{array}{l}37^{\circ} 36^{\prime} \\
37^{\circ} 34^{\prime} \\
\end{array}$ & $\begin{array}{l}148^{\circ} 42^{\prime} \\
149^{\circ} 09^{\prime} \\
\end{array}$ & $\begin{array}{l}- \\
-\end{array}$ & \\
\hline
\end{tabular}

1 SF State Forest, EC East coast, WC West coast.

${ }^{2}$ NSW New South Wales, ACT Australian Capital Territory, Vic. Victoria, Tas. Tasmania.

excluded from the analysis. The block containing the E. nitens and E. globulus control seedlots was not included in the analysis. Data were analysed using ANOVA to test for species, site, and provenance effects, and the interactions species $\times$ site, and provenance (species) $\times$ site. Owing to a combination of the layout, varying numbers of replicates at each site, and uneven number of individuals in each provenance, F-ratios (Satterthwaite-type approximate tests) were obtained using the TEST option within the RANDOM statement in SAS.

The models were simplified where trait classes had some missing values, in order to enable estimation of least-squares means.

Analysis proceeded in three stages:

1. Full data set: Following exploratory analysis, the final model used the fixed terms: site, species, and species $\times$ site interaction, as well as the random terms: provenance (species), provenance (species) $\times$ site, and replicate $\times$ species (site) (Table 4).

2. By Site. Secondly, data for each site were separately analysed to test for species $\times$ provenance effects within each site. The model included the fixed terms: species, provenance, and species $\times$ provenance, and the random terms: provenance (species), and replicate $\times$ provenance (species) (Table 5).
3. By Species. Thirdly, data for each species was analysed separately to test for provenance effects across sites. The test included the fixed terms: provenance, site, and provenance $\times$ site, and the random terms: replicate (site), and provenance $x$ replicate (site) (Table 6).

These analyses were performed using the Proc GLM procedure in SAS 9.1. Tukey's multiple range test was used to indicate significance of difference among sites, species or provenance within species means for all traits.

\section{Results}

The suppression of Eucalypus pseudoglobulus by E. globulus meant that results from only three species (E. globulus, E. maidenii and E. bicostata) are reported here.

\section{Site effects}

Using the full data set, site significantly affected all traits $(p<0.002$, Table 4$)$. The main site effects were $\mathrm{DBH}$ and $\mathrm{HT}$, which were highest at McKinnon for all species (Figure 2a,b). The McKinnon site was by far $(p<0.001)$ the most productive, with DBH of $16.9 \mathrm{~cm}$ compared with Otaua $15.4 \mathrm{~cm}$ and Kawerau $13.2 \mathrm{~cm}$; and $\mathrm{HT}$ at McKinnon of $15.6 \mathrm{~m}$ compared with Otaua $12.2 \mathrm{~m}$ and Kawerau $10.4 \mathrm{~m}$ at age 7 years (Figure 2a,b). Further, McKinnon was ranked first overall for DBH, HT, 
Table 4 Full data set: $p$ values for effects of site, species, provenance within species and their interactions

\begin{tabular}{|c|c|c|c|c|c|c|c|}
\hline \multirow[t]{2}{*}{ Effect } & \multirow[t]{2}{*}{ df } & \multicolumn{6}{|c|}{ Trait $^{1}$} \\
\hline & & DBH & HT & $\mathrm{GCH}$ & $\mathrm{FH}$ & STR & SUR \\
\hline Site & 2 & 0.0002 & $<0.0001$ & $<0.0001$ & 0.002 & 0.002 & $<0.0001$ \\
\hline Species & 2 & $<0.0001$ & $<0.0001$ & $<0.0001$ & $<0.0001$ & 0.001 & $<0.0001$ \\
\hline Species $\times$ site & 4 & 0.7 & 0.6 & 0.3 & 0.03 & $<0.0001$ & 0.004 \\
\hline Provenance (species) & 18 & 0.14 & 0.4 & 0.7 & 0.09 & 0.0031 & 0.4 \\
\hline Provenance $($ species $) \times$ site & 36 & 0.09 & 0.15 & 0.005 & 0.15 & 0.9 & 0.12 \\
\hline Provenance (species) $\times$ replicate (site) & 42 & 0.0002 & $<0.0001$ & $<0.0001$ & 0.02 & 0.003 & 0.005 \\
\hline
\end{tabular}

Replicate (site) effects (not shown) were usually not significant $(p>0.05)$.

${ }^{1}$ Numbers in bold type are significant $(p<0.05)$.

FH, GCH and STR (Table 7). Trait means for Otaua were usually intermediate, except for SUR (highest) and FH (lowest) (Table 7). Kawerau was severely frosted in the first growing season, and this appears to have contributed to its lowest rank for all traits except for $\mathrm{FH}$.

\section{Species effects}

Species differed significantly for all traits $(p=<0.001$; Table 4$)$ and at all sites $(p=<0.05$; Table 5$)$ with the exception of survival at McKinnon, which did not differ significantly by species. Eucalyptus maidenii was significantly better for all traits (Table 7). Eucalyptus bicostata was the lowest-ranked for STR overall, while E. globulus ranked lowest overall for SUR (Table 7). By

Table 5 By Site: significance ( $p$ values) of species and provenance effects for each test site: Northland (Otaua), Bay of Plenty (Kawerau) and Hawke's Bay (McKinnon)

\begin{tabular}{|c|c|c|c|c|}
\hline \multirow[t]{2}{*}{ Trait } & \multirow[t]{2}{*}{ Effect } & \multicolumn{3}{|c|}{ Site $^{1}$} \\
\hline & & Otaua & Kawerau & McKinnon \\
\hline$\overline{\mathrm{DBH}}$ & $\begin{array}{l}\text { Species } \\
\text { Rep } \\
\text { Provenance (species) } \\
\text { Provenance (species) } \times \text { rep }\end{array}$ & $\begin{array}{l}<\mathbf{0 . 0 0 0 1} \\
0.5 \\
\mathbf{0 . 0 0 0 1} \\
0.7\end{array}$ & $\begin{array}{l}<\mathbf{0 . 0 0 0 1} \\
\mathbf{0 . 0 0 7} \\
0.20 \\
0.5\end{array}$ & $\begin{array}{l}<\mathbf{0 . 0 0 0 1} \\
0.5 \\
0.7 \\
0.041\end{array}$ \\
\hline $\mathrm{HT}$ & $\begin{array}{l}\text { Species } \\
\text { Rep } \\
\text { Provenance (species) } \\
\text { Provenance (species) } \times \text { rep }\end{array}$ & $\begin{array}{l}<\mathbf{0 . 0 0 0 1} \\
0.5 \\
\mathbf{0 . 0 1 3} \\
\mathbf{0 . 0 1 1}\end{array}$ & $\begin{array}{l}<\mathbf{0 . 0 0 0 1} \\
\mathbf{0 . 0 0 4} \\
0.5 \\
0.7\end{array}$ & $\begin{array}{l}<\mathbf{0 . 0 0 0 1} \\
0.12 \\
0.8 \\
0.8\end{array}$ \\
\hline $\mathrm{GCH}$ & $\begin{array}{l}\text { Species } \\
\text { Rep } \\
\text { Provenance (species) } \\
\text { Provenance (species) } \times \text { rep }\end{array}$ & $\begin{array}{l}<\mathbf{0 . 0 0 0 1} \\
0.2 \\
0.3 \\
\mathbf{0 . 0 0 1}\end{array}$ & $\begin{array}{l}<\mathbf{0 . 0 0 0 1} \\
\mathbf{0 . 0 0 1} \\
0.6 \\
0.26\end{array}$ & $\begin{array}{l}\mathbf{0 . 0 0 3} \\
0.7 \\
0.16 \\
0.9\end{array}$ \\
\hline $\mathrm{FH}$ & $\begin{array}{l}\text { Species } \\
\text { Rep } \\
\text { Provenance (species) } \\
\text { Provenance (species) × rep }\end{array}$ & $\begin{array}{l}<\mathbf{0 . 0 0 0 1} \\
0.069 \\
\mathbf{0 . 0 0 3} \\
0.8\end{array}$ & $\begin{array}{l}<\mathbf{0 . 0 0 0 1} \\
0.7 \\
0.16 \\
0.98\end{array}$ & $\begin{array}{l}<\mathbf{0 . 0 0 0 1} \\
0.18 \\
0.6 \\
\mathbf{0 . 0 1 4}\end{array}$ \\
\hline STR & $\begin{array}{l}\text { Species } \\
\text { Rep } \\
\text { Provenance (species) } \\
\text { Provenance (species) } \times \text { rep }\end{array}$ & $\begin{array}{l}0.009 \\
0.2 \\
0.6 \\
0.5\end{array}$ & $\begin{array}{l}<\mathbf{0 . 0 0 0 1} \\
0.99 \\
0.5 \\
0.098\end{array}$ & $\begin{array}{l}\mathbf{0 . 0 4 9} \\
0.5 \\
\mathbf{0 . 0 1 3} \\
0.96\end{array}$ \\
\hline SUR & $\begin{array}{l}\text { Species } \\
\text { Rep } \\
\text { Provenance (species) } \\
\text { Provenance (species) × rep }\end{array}$ & $\begin{array}{l}\mathbf{0 . 0 0 6} \\
0.099 \\
0.069 \\
0.96\end{array}$ & $\begin{array}{l}<\mathbf{0 . 0 0 0 1} \\
0.13 \\
0.2 \\
0.7\end{array}$ & $\begin{array}{l}0.6 \\
0.7 \\
0.4 \\
0.15\end{array}$ \\
\hline
\end{tabular}

${ }^{1}$ Numbers in bold type are significant $(p<0.05)$. comparison, the control seedlot of E. nitens had grown slightly faster than the E. maidenii (DBH $15.8 \mathrm{~cm}$ and $15.2 \mathrm{~cm}$, HT $13.9 \mathrm{~m}$ and $12.7 \mathrm{~m}$ respectively), the faster growth of $E$. nitens is likely due in part to the superior health of its foliage compared to the other species tested (Table 7).

\section{Species $\times$ site effects}

There was no species $\times$ site interaction for the traits $\mathrm{DBH}, \mathrm{HT}$ and GCH (Table 4). Species $\times$ site interactions were observed for FH $(\mathrm{P}<0.05)$, and STR and SUR (both $\mathrm{p}<0.001$, Table 4), which indicates that analysis within species is necessary to interpret these results. A similar dynamic occurs for STR, where for E. bicostata straightness is atypically high at Otaua, and low at Kawerau (Figure 2d). The species $\times$ site interaction involving SUR appears to involve a combination of the poor survival of E. globulus at Kawerau, and the change in rank of E. globulus and E. bicostata at McKinnon among provenances within species, and site interaction effects.

Provenance (species) and provenance (species) $\times$ site effects There was no provenance (species) $\times$ site interaction for any trait of E. maidenii (Table 6). This was mostly the case for E. globulus too, except for the occurrence of a low level $(\mathrm{p}=0.016)$ provenance $\times$ site interaction for $\mathrm{HT}$, probably due to the change in rank (first at Otaua, last at McKinnon) of the Clarke Island provenance (details not shown). Significant provenance $\times$ site interaction occurred for most traits within E. bicostata (Table 6).

Provenances did vary significantly within E. bicostata for all traits $(\mathrm{p}<0.05)$ except survival $(\mathrm{p}=0.055)$ (Table 6). The E. bicostata provenance Wollemi had the highest $\mathrm{DBH}$ and $\mathrm{FH}$ of all seven provenances at the Otaua site (Table 8), but was much closer to the mean at McKinnon and Kawerau. The other interactions within E. bicostata appear to be due to changes in scale, with the Benalla provenance having higher survival at McKinnon, the Mansfield provenance recording lower DBH and HT figures at McKinnon. Lastly, while $\mathrm{GCH}$ was tightly grouped for the E. bicostata provenances at Kawerau, there was greater variaation among provenances, and 
Table 6 By Species: significance ( $p$ values) of site and provenance effects for each species

\begin{tabular}{|c|c|c|c|c|c|c|c|}
\hline \multirow[t]{3}{*}{ Trait } & \multirow[t]{3}{*}{ Source } & \multicolumn{6}{|c|}{ Species } \\
\hline & & \multicolumn{2}{|c|}{ E. maidenii } & \multicolumn{2}{|c|}{ E. globulus } & \multicolumn{2}{|c|}{ E. bicostata } \\
\hline & & df & $p$ & df & $p$ & df & $p$ \\
\hline$\overline{\mathrm{DBH}}$ & $\begin{array}{l}\text { Site } \\
\text { Replicate(site) } \\
\text { Provenance } \\
\text { Provenance } \times \text { site } \\
\text { Provenance } \times \text { replicate (site) } \\
\text { Error }\end{array}$ & $\begin{array}{c}2 \\
14 \\
6 \\
12 \\
82 \\
218\end{array}$ & $\begin{array}{l}\mathbf{0 . 0 0 6} \\
0.1 \\
0.6 \\
0.2 \\
0.5\end{array}$ & $\begin{array}{c}2 \\
13 \\
6 \\
12 \\
51 \\
103\end{array}$ & $\begin{array}{l}0.1 \\
0.2 \\
0.9 \\
0.2 \\
0.4\end{array}$ & $\begin{array}{c}2 \\
14 \\
6 \\
8 \\
63 \\
140\end{array}$ & $\begin{array}{l}\mathbf{0 . 0 0 4} \\
0.07 \\
<\mathbf{0 . 0 0 0 1} \\
\mathbf{0 . 0 0 4} \\
0.7\end{array}$ \\
\hline $\mathrm{HT}$ & $\begin{array}{l}\text { Site } \\
\text { Replicate }(\text { site) } \\
\text { Provenance } \\
\text { Provenance } \times \text { site } \\
\text { Provenance } \times \text { replicate (site) } \\
\text { Error }\end{array}$ & $\begin{array}{c}2 \\
14 \\
6 \\
12 \\
75 \\
136\end{array}$ & $\begin{array}{l}\mathbf{0 . 0 0 1} \\
<\mathbf{0 . 0 0 0 1} \\
0.8 \\
0.8 \\
0.95\end{array}$ & $\begin{array}{c}2 \\
13 \\
6 \\
11 \\
43 \\
61\end{array}$ & $\begin{array}{l}<\mathbf{0 . 0 0 0 1} \\
\mathbf{0 . 0 0 7} \\
0.1 \\
\mathbf{0 . 0 1 6} \\
0.9\end{array}$ & $\begin{array}{c}2 \\
14 \\
6 \\
8 \\
47 \\
86\end{array}$ & $\begin{array}{l}0.002 \\
0.001 \\
0.007 \\
0.2 \\
0.3\end{array}$ \\
\hline $\mathrm{GCH}$ & $\begin{array}{l}\text { Site } \\
\text { Replicate }(\text { site) } \\
\text { Provenance } \\
\text { Provenance } \times \text { site } \\
\text { Provenance } \times \text { replicate (site) } \\
\text { Error }\end{array}$ & $\begin{array}{c}2 \\
14 \\
6 \\
12 \\
75 \\
134\end{array}$ & $\begin{array}{l}\mathbf{0 . 0 0 2} \\
<\mathbf{0 . 0 0 0 1} \\
0.4 \\
0.1 \\
0.7\end{array}$ & $\begin{array}{c}2 \\
13 \\
6 \\
11 \\
40 \\
59\end{array}$ & $\begin{array}{l}<\mathbf{0 . 0 0 0 1} \\
0.3 \\
0.9 \\
0.1 \\
0.6\end{array}$ & $\begin{array}{c}2 \\
14 \\
6 \\
8 \\
47 \\
84\end{array}$ & $\begin{array}{l}0.001 \\
<0.0001 \\
0.020 \\
\mathbf{0 . 0 0 2} \\
0.99\end{array}$ \\
\hline $\mathrm{FH}$ & $\begin{array}{l}\text { Site } \\
\text { Replicate }(\text { site) } \\
\text { Provenance } \\
\text { Provenance } \times \text { site } \\
\text { Provenance } \times \text { replicate (site) } \\
\text { Error }\end{array}$ & $\begin{array}{c}2 \\
14 \\
6 \\
12 \\
82 \\
209\end{array}$ & $\begin{array}{l}\mathbf{0 . 0 0 6} \\
0.05 \\
0.2 \\
0.2 \\
0.8\end{array}$ & $\begin{array}{c}2 \\
13 \\
6 \\
12 \\
51 \\
103\end{array}$ & $\begin{array}{l}0.4 \\
\mathbf{0 . 0 2 4} \\
0.5 \\
0.4 \\
0.4\end{array}$ & $\begin{array}{c}2 \\
14 \\
6 \\
8 \\
63 \\
136\end{array}$ & $\begin{array}{l}\mathbf{0 . 0 2 7} \\
0.2 \\
\mathbf{0 . 0 0 1} \\
\mathbf{0 . 0 0 1} \\
0.99\end{array}$ \\
\hline STR & $\begin{array}{l}\text { Site } \\
\text { Replicate(site) } \\
\text { Provenance } \\
\text { Provenance } \times \text { site } \\
\text { Provenance } \times \text { replicate (site) } \\
\text { Error }\end{array}$ & $\begin{array}{c}2 \\
14 \\
6 \\
12 \\
82 \\
217\end{array}$ & $\begin{array}{l}0.7 \\
0.072 \\
0.6 \\
0.3 \\
0.99\end{array}$ & $\begin{array}{c}2 \\
13 \\
6 \\
12 \\
51 \\
103\end{array}$ & $\begin{array}{l}\mathbf{0 . 0 2 7} \\
0.2 \\
1.0 \\
0.5 \\
0.8\end{array}$ & $\begin{array}{c}2 \\
14 \\
6 \\
8 \\
63 \\
140\end{array}$ & $\begin{array}{l}\mathbf{0 . 0 0 0 2} \\
\mathbf{0 . 0 0 6} \\
\mathbf{0 . 0 0 0 3} \\
0.93 \\
0.3\end{array}$ \\
\hline SUR & $\begin{array}{l}\text { Site } \\
\text { Replicate(site) } \\
\text { Provenance } \\
\text { Provenance } \times \text { site } \\
\text { Provenance } \times \text { replicate (site) } \\
\text { Error }\end{array}$ & $\begin{array}{c}2 \\
14 \\
6 \\
12 \\
84 \\
305\end{array}$ & $\begin{array}{l}\mathbf{0 . 0 0 1} \\
0.3 \\
0.4 \\
0.6 \\
0.5\end{array}$ & $\begin{array}{c}2 \\
14 \\
6 \\
12 \\
77 \\
286\end{array}$ & $\begin{array}{l}<\mathbf{0 . 0 0 0 1} \\
\mathbf{0 . 0 2 7} \\
0.3 \\
0.09 \\
0.7\end{array}$ & $\begin{array}{c}2 \\
14 \\
6 \\
8 \\
69 \\
256\end{array}$ & $\begin{array}{l}\mathbf{0 . 0 0 4} \\
\mathbf{0 . 0 0 0 3} \\
0.055 \\
0.2 \\
0.96\end{array}$ \\
\hline
\end{tabular}

${ }^{1}$ Numbers in bold type are significant $(p<0.05)$.

higher $\mathrm{GCH}$ provenance means at Otaua and McKinnon (Table 8). Traits that are affected by competition, or traits that are subjectively scored, may provide significance levels approaching unity. This is the case for several traits at the provenance $\times$ replicate (site) level.

\section{Discussion}

Based on previous studies in various parts of the world, the relative performance of the four blue gum species, Eucalyptus bicostata, E. globulus, E. maidenii and E. pseudoglobulus, is expected to be related to the fit between the environmental parameters of both the collection (provenence) and test sites (e.g. Dutkowski and Potts 1999, Jordan et al., 1993). Given the equivalent latitude of New Zealand's North Island to the core distributions of E. bicostata and E. maidenii then those species might be expected to perform better than $E$. globulus, the core of which comes from much higher latitudes (Jordan et al., 1993) (Figure 1).

The lack of a species $\times$ site effect for both growth variables (DBH and height), and the consistent high performance of $E$. maidenii, suggest that all three test locations are well suited to that species but not to the other three blue gum species. However, the three test sites varied greatly in early productivity, for reasons that could include: varying rainfall, nutrient availability or evapotranspiration among the sites, out-of-season frosts, and/or establishment practice. The three trial sites all had different pre-plant sprays, for example; broadcast at McKinnon, spot mounding and spraying at Otaua, and ripping and mounding at Kawerau followed by pre-plant spray. Regardless of the variation in environmental conditions among the three test sites, the ranking of the species was the same at each site. This result suggests 


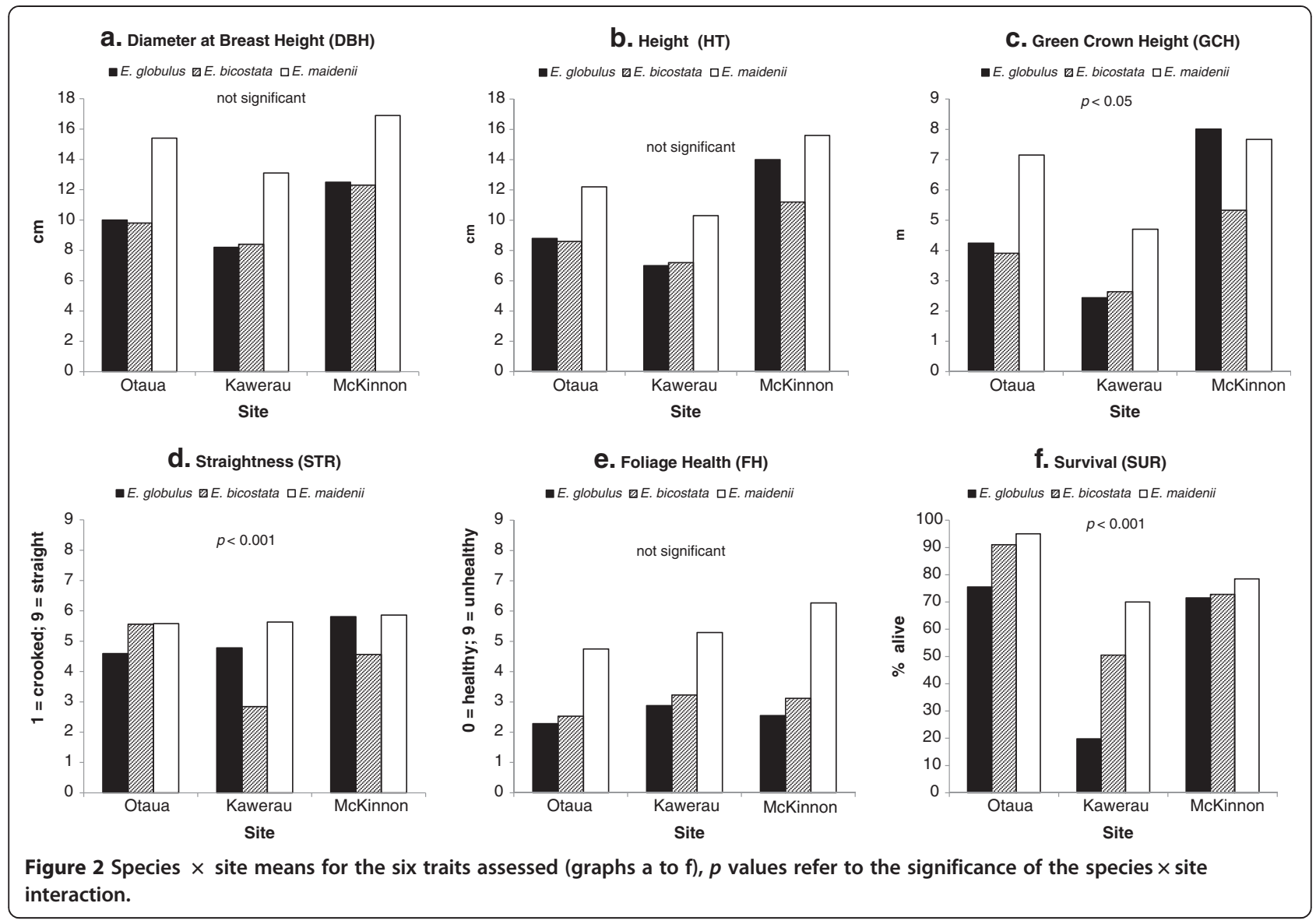

that the climate of all three New Zealand test sites more closely matches the natural climate range of E. maidenii than those of the other species.

Warm, wet summer weather experienced at the three test locations is conductive to fungal Mycosphaerella leaf disease outbreaks (Park, 1988; Carnegie, 1994) that cause leaf necrosis and defoliation resulting in reduced growth (Park and Keane, 1982). Of the eucalypt species assessed at the three test locations only E. maidenii (with the exception of the Wollemi provenance of E. bicostata which

Table 7 Least-squares means by species and by site

\begin{tabular}{llllllll}
\hline \multirow{2}{*}{ Effect } & Levels & \multicolumn{6}{c}{ Trait } \\
\cline { 3 - 8 } & & DBH & HT & GCH & STR & FH & SUR \\
\hline Species & E. maidenii & $15.2 \mathrm{a}$ & $12.7 \mathrm{a}$ & $6.5 \mathrm{a}$ & $5.7 \mathrm{a}$ & $5.4 \mathrm{a}$ & $0.81 \mathrm{a}$ \\
& E. globulus & $10.4 \mathrm{~b}$ & $9.9 \mathrm{~b}$ & $4.9 \mathrm{~b}$ & $5.1 \mathrm{~b}$ & $2.6 \mathrm{~b}$ & $0.57 \mathrm{~b}$ \\
& E. bicostata & $10.2 \mathrm{~b}$ & $9.0 \mathrm{~b}$ & $4.0 \mathrm{~b}$ & $4.3 \mathrm{c}$ & $3.0 \mathrm{~b}$ & $0.71 \mathrm{a}$ \\
& E. nitens & 15.8 & 13.9 & 8.3 & 3.3 & 7.1 & 0.81 \\
Site & & & & & & & \\
& Otaua & $11.7 \mathrm{~b}$ & $9.9 \mathrm{~b}$ & $5.1 \mathrm{~b}$ & $5.2 \mathrm{a}$ & $3.2 \mathrm{~b}$ & $0.87 \mathrm{a}$ \\
& Kawerau & $10.1 \mathrm{C}$ & $8.2 \mathrm{~b}$ & $3.3 \mathrm{c}$ & $4.4 \mathrm{~b}$ & $3.8 \mathrm{a}$ & $0.47 \mathrm{c}$ \\
& McKinnon & $13.9 \mathrm{a}$ & $13.6 \mathrm{a}$ & $7.0 \mathrm{a}$ & $5.4 \mathrm{a}$ & $4.0 \mathrm{a}$ & $0.75 \mathrm{~b}$
\end{tabular}

Demonstration plots of $E$. nitena included as a comparison.

${ }^{1}$ Differences for $E$. nitens were not tested against blue gum species means. Values for species means (within each trait group) when followed by the same letter are not significantly different $(p>0.05)$. grew well compared to the other provenances of this species tested) originate from regions that experience uniform rainfall throughout the year (Boland et al., 1992). Having evolved in regions where Mycosphaerella is more severe, E. maidenii is likely to have undergone more intense natural selection to such pathogens making them more resistant. This theory would explain the better foliage health and growth rate experienced by $E$. maidenii assessed in this study. Other studies have noted similar associations between high rainfall and warm temperatures and lower susceptibility to Mycosphaerella spp. in provenances of E. globulus (Dungey et al., 1997; Carnegie, 1994).

In addition to Mycosphaerella resistance, the three species (Eucalyptus maidenii, E. globulus and E. bicostata) may differ in their physiological adaptation to the test site conditions. For example, Anekonda et al. (1999) reported differences in respiratory metabolism between two $\mathrm{Eu}$ calyptus subgenera. It is presumed that physiological differences may be detected among species within subgenera, particularly where the species are related and are distributed along a climatic cline. Such variation is suggested by the latitudinal cline in lignin content among blue gum species (Rencoret et al., 2007), and 
Table 8 Provenance least-squares means for Eucalyptus bicostata traits at each site

\begin{tabular}{|c|c|c|c|c|c|c|c|c|c|}
\hline \multirow[t]{2}{*}{ Provenance } & \multicolumn{3}{|c|}{$\mathrm{DBH}$} & \multicolumn{3}{|c|}{$\mathrm{HT}$} & \multicolumn{3}{|c|}{$\mathrm{GCH}$} \\
\hline & Otaua & Kawerau & McKinnon & Otaua & Kawerau & McKinnon & Otaua & Kawerau & McKinnon \\
\hline Canberra & - & 10.1 & - & - & 7.9 & - & - & 2.8 & - \\
\hline Burrinjuck & $8.3 \mathrm{bc}$ & 8.4 & 13.6 & $8.2 b c$ & 6.9 & 12.0 & $5.0 \mathrm{ab}$ & 2.2 & 4.3 \\
\hline Tumbarumba & - & 6.7 & - & - & 6.9 & - & - & 2.7 & - \\
\hline Wollemi & $14.1 \mathrm{a}$ & 10.0 & 12.0 & $9.7 \mathrm{a}$ & 7.4 & 10.7 & $2.8 \mathrm{C}$ & 2.6 & 5.3 \\
\hline Benalla & $9.5 \mathrm{~b}$ & 9.6 & 12.4 & $9.2 \mathrm{ab}$ & 8.1 & 11.3 & $5.4 \mathrm{a}$ & 2.5 & 6.2 \\
\hline Kiewa & $7.0 \mathrm{C}$ & 6.2 & 10.0 & $7.3 \mathrm{c}$ & 6.3 & 11.5 & $3.8 \mathrm{abc}$ & 3.0 & 5.1 \\
\hline Mansfield & $7.3 \mathrm{bc}$ & 8.5 & 10.8 & $7.2 \mathrm{C}$ & 7.0 & 8.5 & $3.5 b c$ & 2.3 & 4.2 \\
\hline$p$ value & $<0.0001$ & 0.0501 & 0.36 & $<0.0001$ & 0.35 & 0.4 & $<0.0001$ & 0.8 & 0.41 \\
\hline \multirow[t]{2}{*}{ Provenance } & \multicolumn{3}{|c|}{$\mathrm{FH}$} & \multicolumn{3}{|c|}{ STR } & \multicolumn{3}{|c|}{ SUR } \\
\hline & Otaua & Kawerau & McKinnon & Otaua & Kawerau & McKinnon & Otaua & Kawerau & McKinnon \\
\hline Canberra & - & 4.0 & - & - & 4.2 & - & - & $0.67 \mathrm{a}$ & - \\
\hline Burrinjuck & $1.6 \mathrm{~b}$ & 3.2 & 3.1 & $5.2 \mathrm{~b}$ & 2.9 & 3.4 & 0.88 & $0.56 \mathrm{ab}$ & 0.61 \\
\hline Tumbarumba & - & 2.7 & - & - & 2.3 & - & - & $0.30 \mathrm{~b}$ & - \\
\hline Wollemi & $4.5 \mathrm{a}$ & 3.6 & 3.3 & $5.7 \mathrm{ab}$ & 2.6 & 4.2 & 0.90 & $0.67 \mathrm{a}$ & 0.77 \\
\hline Benalla & $1.9 \mathrm{~b}$ & 3.3 & 3.1 & $7.0 \mathrm{a}$ & 3.9 & 6.3 & 0.89 & $0.33 a b$ & 0.88 \\
\hline Kiewa & $2.0 \mathrm{~b}$ & 2.1 & 2.8 & $5.7 \mathrm{ab}$ & 3 & 4.8 & 0.89 & $0.52 \mathrm{ab}$ & 0.69 \\
\hline Mansfield & $1.9 \mathrm{~b}$ & 3.7 & 2.9 & $4.9 \mathrm{~b}$ & 2.3 & 4.3 & 1.01 & $0.51 \mathrm{ab}$ & 0.70 \\
\hline$p$ value & $<0.0001$ & 0.157 & 0.944 & 0.006 & 0.055 & 0.049 & 0.68 & 0.031 & 0.47 \\
\hline
\end{tabular}

Values (within each group) followed by the same letter are not significantly different $(p>0.05)$.

the higher tolerance of inter-tree competition exhibited by E. maidenii versus E. globulus and other eucalypt species in even-spaced monoculture (Low \& Shelbourne, 1999). The functional adaptation of genotypes to latitude is suggested within E. globulus, whereby density, and pulp yield show particular patterns of variation with latitude (Stackpole et al., 2010b). The broad latitudinal range of $E$. bicostata encompassed both the winter and uniform rainfall zones. The most northern $E$. bicostata provenance (Wollemi) in the present study occurred in a uniform rainfall zone, and displayed the best growth rate and foliage health of the seven E. bicostata provenences tested in the New Zealand test sites. This result is similar to the findings of (Komakech et al. 2009), although the causality of this association remains to be demonstrated.

The superior growth of E. maidenii found in this study compared with the other three blue gum species reflects the findings of (Shelbourne et al. 2002) from longerterm large-plot comparisons with E. globulus. This trait, plus the reasonable pulping qualities (Kibblewhite et al., 2000), high density (McKinley et al., 2002), and the favourable silvicultural characteristics of $E$. maidenii, seem to indicate the suitability of this species for projects where biomass, carbon or nutrient accumulation are contributing objectives. Similarly, the density of blue gum species varies; with $E$. maidenii reported at $550 \mathrm{~kg} \mathrm{~m}^{-3}$ (McKinley et al., 2002), well in excess of published density of E. globulus $\left(470 \mathrm{~kg} \mathrm{~m}^{-3}\right)$ from that and other studies (Miranda et al., 2001; Stackpole et al., 2010a).

While the results here have shown clear preference to E. maidenii, it is known that genotypes grown in mixtures do not always reflect the performance of genotypes in large plots (Fasoula and Fasoula 1997; Retief et al., 2001; Stanger et al. 2011). However, (Shelbourne et al. 2002) also found E. maidenii to be among the best performers in large-plot trials. Therefore consider that results are sufficiently robust to: (a) conclude this species outperforms the other blue gums tested; and (b) to recommend this species as suitable for further domestication for New Zealand conditions. In order to undertake this recommendation, further E. maidenii introductions from natural origins would be required. The lack of a significant provenance effect in the $E$. maidenii in these trials suggests that a collection based on range-wide open-pollinated families from welldefined populations is possible. A sampling strategy that obtains 200-400 family seedlots from across the provenances, and selection for key pulp production traits including density and pulp yield is recommended. Such a venture could be established in partnership with Australian co-operators with a view to characterising genotype $\mathrm{x}$ environment interaction.

The site at Kawerau was severely frosted in the first growing season with around three-quarters of the trees losing their foliage. The E. nitens plots suffered only minor damage, and E. maidenii appeared to be slightly less affected than E. bicostata, E. globulus, or E. pseudoglobulus. The majority of the plants re-sprouted and grew to crop trees, but this incident serves as a warning to planting these species in non-coastal regions of New Zealand. Relatively few frosts are predicted per year at Kawerau ( 15, Table 2) compared to the conditions experienced in the species' natural ranges (Table 1). 
However, it is likely that New Zealand is more prone to out-of-season frosts compared to Australia due to its maritime climate, and these frost events, rather than total number of frosts, are more damaging to young plants. Traditionally, small seedlings have been planted out in winter (these trials included) but planting in late spring is likely to improve survival. Spring-planted seedlings are likely to grow sufficiently in the first season to survive any frosts that occur the following year.

\section{Conclusion}

Eucalyptus maidenii appears to be the best-suited blue gum species to sites in the northern half of New Zealand's North Island. This may be due to similarities in climatic characteristics among the trial sites and natural E. maidenii populations. The relatively close grouping of natural provenances and variation in $E$. maidenii are unlikely to affect which provenances should be used for the selection of genetic material for further development in New Zealand, except that as wide a collection as possible would help ensure a broad genetic base.

Adaptation to uniform rainfall climate is also evident in the superior performance of the northerly Wollemi provenance of E. bicostata in the present trial series.

\section{Endnotes}

aThe range of Eucalyptus bicostata extends to sites in northern New South Wales (e.g. outlier populations in the Walcha area) that experience a third type of rainfall pattern, summer maximum rainfall distribution. This type of site was not represented in the New Zealand trials.

${ }^{\mathrm{b}}$ where $0=$ dead or dying, $1=$ severe damage, $2=$ bad damage and tree obviously suffering, $3=$ moderate-tobad damage, $4=$ moderate damage, but above average, $5=$ moderate damage, but below average, $6=$ low-moderate damage, $7=$ damage clear but low, $8=$ some damage evident, $9=$ healthy.

\section{Competing interests}

The authors declare that they have no competing interests.

\section{Authors' contributions}

HD drafted the first version of this manuscript, re-analysed the data, based on a technical report written by RMCC, who also organised and helped measure the trials. DS again re-analysed the data and drafted the second version of this manuscript which was submitted. RB and HD provided guidance on the context and content of the manuscript and SK revised re-analysed where appropriate and helped us to create the final version, addressing reviewers comments. All authors were involved in technical discussions contributing to the production of the manuscript with the other authors during this research. All authors read and approved the final manuscript.

\section{Acknowledgements}

The authors acknowledge members of the Eucalypt Co-operative (now a part of Future Forest Research), funding from New Species C04X0303 and Diverse Forests C04X0805 contracts through the Foundation for Research Science and Technology; the staff of PF Olsen Ltd for assisting with establishment and management, the late lan Nicholas for help with the assessment and Marie Heaphy for supplying climatic data for the three New Zealand trial sites.

\section{Author details}

'Scion, 49 Sala Street, Private Bag, 3020, Rotorua, New Zealand. ${ }^{2} 250$ Main Rd, R D 1 Wakefield, Brightwater, New Zealand.

Received: 3 May 2013 Accepted: 3 May 2013

Published: 24 June 2013

\section{References}

Almeida, MH, Pereira, H, Miranda, I, \& Tome, M. (1995). Provenance trials of Eucalyptus globulus Labill. in Portugal. Hobart, Australia: CRC for Temperate Hardwood Forestry.

Anekonda, TS, Criddle, RS, Bacca, M, \& Hansen, LD. (1999). Contrasting adaptation of two Eucalyptus subgenera is related to differences in respiratory metabolism. Functional Ecology, 13(5), 675-682.

Boland, DJ, Brooker, MIH, Chippendale, GM, Hall, N, Hyland, BPM, Johnston, RD, Kleinig, DA, \& Turner, JD. (1992). Forest Trees of Australia. Melbourne, Australia: CSIRO Publishing.

Booth, T. (2005). Environment, species selection and productivity prediction. In S Nambiar \& I Ferguson (Eds.), New Forests: Wood Production and Environmental Services (pp. 5-23). Collingwood, Victoria, Australia: CSIRO Publishing.

Brooker, M. (2000). A new classification of the genus Eucalyptus L'Her. (Myrtaceae). Australian Systematic Botany, 13, 79-148.

Bureau of Meteorology. (2012). Australian seasonal rainfall zones. Accessed from http://www.bom.gov.au/climate/environ/other/IDCJCM0002_aus_seas_zones. shtml, 14th September 2012

Carnegie, AJ, Keane, PJ, Ades, PK, \& Smith, IW. (1994). Variation in susceptibility of Eucalyptus globulus provenances to Mycosphaerella leaf disease. Canadian Journal of Forest Research, 24, 1751-1757.

Cotterill, P, \& Brolin, A. (1997). Improving Eucalyptus wood, pulp and paper quality by genetic selection (pp. 1-14). Salvador, Brazil.

Díez, J. (2005). Invasion biology of Australian ectomycorrhizal fungi introduced with eucalypt plantations into the Iberian Peninsula. Biological Invasions, 7(1), $3-15$.

Dungey, HS, Potts, BM, Carnagie, AJ, \& Ades, PK. (1997). Mycosphaerella leaf disease: genetic variation in damage to Eucalyptus nitens, Eucalyptus globulus, and their F1 hybrid. Canadian Journal of Forest Research, 27, 750-759.

Dutkowski, GW, \& Potts, BM. (1999). Geographic patterns of genetic variation in Eucalyptus globulus ssp globulus and a revised racial classification. Australian Journal of Botany, 47, 237-263.

Eldridge, K, Davidson, J, Harwood, C, \& Van Wyk, G. (1993). Eucalypt Domestication and Breeding. Oxford University Press: Oxford.

Fasoula, DA, \& Fasoula, VA. (1997). Competitive ability and plant breeding. Plant Breeding Reviews, 14, 89-138.

Hood, I, Gardner, J, \& Kimberley, M. (2002). Variation among eucalypt species in early susceptibility to leaf spot fungi Phaeophleospora eucalypti and Mycosphaerella ssp. New Zealand Journal of Forestry Science, 32(2), 235-255.

Jones, RC, Steane, DA, Potts, BM, \& Vaillancourt, RE. (2002). Microsatellite and morphological analysis of Eucalyptus globulus populations. Canadian Journal of Forest Research, 32, 59-66

Jordan, GJ, Potts, BM, Kirkpatrick, JB, \& Gardiner, C. (1993). Variation in the Eucalyptus globulus complex revisited. Australian Journal of Botany, 41, 763-785.

Kibblewhite, RP, Johnson, BI, \& Shelbourne, CJA. (2000). Kraft pulp qualities of Eucalyptus nitens, E. globulus, and E. maidenii, at ages 8 and 11 years. New Zealand Journal of Forestry Science, 30(3), 447-457.

Kirkpatrick, JB. (1975). The numerical intraspecific taxonomy of Eucalyptus globulus Labill. (Myrtaceae). Botanical Journal of the Linnean Society, 69, 89-104.

Komakech, C, Swain, T, \& Fossey, A. (2009). Growth potential of Eucalyptus globulus subsp. bicostata provenances for the mid-altitude summer rainfall regions of South Africa. Southern Forests, 71(1), 1-9.

Lanfranco, D, \& Dungey, HS. (2001). Insect damage in Eucalyptus: a review of plantations in Chile. Austral Ecology, 26(5), 477-481.

Low, CB, \& Shelbourne, CJA. (1999). Performance of Eucalyptus globulus, E. maidenii, E. nitens, and other eucalypts in Northland and Hawke's Bay at ages 7 and 11 years. New Zealand Journal of Forestry Science, 29(2), 274-288.

McKinley, RB, Shelbourne, CJA, Low, CB, Penellum, B, \& Kimberley, MO. (2002). Wood properties of young Eucalyptus nitens, E. globulus, and E. maidenii in Northland, New Zealand. New Zealand Journal of Forestry Science, 32(3), 334-356. 
Miranda, I, \& Pereira, H. (2001). Provenance effect on wood chemical composition and pulp yield for Eucalyptus globulus Labill. Appita Journal, 54(4), 347-351.

Miranda, I, Almeida, MH, \& Pereira, H. (2001). Provenance and site variation of wood density in Eucalyptus globulus Labill,, at harvest age and its relation to a nondestructive early assessment. Forest Ecology and Management, 149(1-3), 235-240.

National Institute of Water and Atmospheric Research. (2011). Retrieved from www.niwa.co.nz.

Nesbitt, K, Potts, BM, Vaillancourt, RE, West, A, \& Reid, J. (1995). Partitioning and distribution of RAPD variation in a forest tree species Eucalyptus globulus (Myrtaceae). Heredity, 74, 628-937.

Park, RF. (1988). Epidemiology of Mycosphaerella nubilosa and M. cryptica on Eucalyptus spp. in south-eastern Australia. Transactions of the British Mycological Society, 91, 261-266.

Park, RF, \& Keane, PJ. (1982). Leaf diseases of Eucalyptus associated with Mycosphaerella species. Transactions of the British Mycological Society, 79, 101-115.

Potts, BM, Vaillancourt, RE, Jordan, G, Dutkowski, GW, Costa e Silva, J, McKinnon, GE, Steane, DA, Volker, P, Lopez, G, Apiolaza, L, Li, Y, Marques, C, \& Borralho, NMG. (2004). Exploration of the Eucalyptus globulus gene pool. Aviero, Portugal: IUFRO.

Rencoret, J, Gutierrez, A, \& del Rio, J. (2007). Lipid and lignin composition of woods from different eucalypt species. Holzforschung, 61(2), 165-174.

Retief, ECL, Stanger, TK, \& Galloway, GM. (2001). Early results from a trial to test the effect of plot design on Eucalyptus hybrid clonal ranking in coastal Zululand, South Africa. In Proceedings of IUFRO Working Party 2.08.03 conference, 'Developing the Eucalypt of the Future'. Valdivia, Chile. 10-15 September 2001.

Shelbourne, CJA, Bulloch, BT, Low, CB, \& McConnochie, RM. (2002). Performance to age 22 years of 49 eucalypts in the Wairarapa District, New Zealand, and review of results from other trials. New Zealand Journal of Forestry Science, 32(2).

Stackpole, DJ, Vaillancourt, RE, de Aguigar, M, \& Potts, BM. (2010a). Age trends in genetic parameters for growth and wood density in Eucalyptus globulus. Tree Genetics and Genomes, 6, 179-193.

Stackpole, DJ, Vaillancourt, RE, Downes, G, Harwood, CE, \& Potts, BM. (2010b). Genetic control of kraft pulp yield in Eucalyptus globulus. Canadian Journal of Forest Research, 50(5), 917-927.

Stanger, TK, Galloway, GM, \& Retief, ECL. (2011). Final results from a trial to test the effect of plot size on Eucalyptus hybrid clonal ranking in coastal Zululand, South Africa. Southern Forests, 73, 131-135.

Vaillancourt, RE, Boomsma, D, \& Nicolle, D. (2001). A disjunct population of Eucalyptus globulus ssp. bicostata from South Australia. Transaction of the Royal Society of South Australia, 125, 65-68.

Volker, P, \& Orme, K. (1988). Provenance trials of Eucalyptus globulus and related species in Tasmania. Australian Forestry, 51(4), 257-265.

doi:10.1186/1179-5395-43-6

Cite this article as: Stackpole et al: Comparative performance of

Eucalyptus bicostata, E. globulus, E. maidenii and E. pseudoglobulus on three northern New Zealand sites. New Zealand Journal of Forestry Science 2013 43:6.

\section{Submit your manuscript to a SpringerOpen ${ }^{\circ}$ journal and benefit from:}

- Convenient online submission

- Rigorous peer review

- Immediate publication on acceptance

- Open access: articles freely available online

- High visibility within the field

- Retaining the copyright to your article

Submit your next manuscript at $\gg$ springeropen.com 